University of Nebraska - Lincoln

DigitalCommons@University of Nebraska - Lincoln

\title{
Surface facial electromyography, skin conductance, and self- reported emotional responses to light- and season-relevant stimuli in seasonal affective disorder
}

\author{
Kathryn Tierney Lindsey \\ Uniformed Services University of the Health Sciences \\ Kelly J. Rohan \\ University of Vermont, kelly.rohan@uvm.edu \\ Kathryn A. Roecklein \\ University of Pittsburgh \\ Jennifer N. Mahon \\ University of Vermont
}

Follow this and additional works at: https://digitalcommons.unl.edu/usuhs

Part of the Medicine and Health Sciences Commons

\begin{abstract}
Lindsey, Kathryn Tierney; Rohan, Kelly J.; Roecklein, Kathryn A.; and Mahon, Jennifer N., "Surface facial electromyography, skin conductance, and self-reported emotional responses to light- and season-relevant stimuli in seasonal affective disorder" (2011). Uniformed Services University of the Health Sciences. 64. https://digitalcommons.unl.edu/usuhs/64
\end{abstract}

This Article is brought to you for free and open access by the U.S. Department of Defense at DigitalCommons@University of Nebraska - Lincoln. It has been accepted for inclusion in Uniformed Services University of the Health Sciences by an authorized administrator of DigitalCommons@University of Nebraska Lincoln. 
Research report

\title{
Surface facial electromyography, skin conductance, and self-reported emotional responses to light- and season-relevant stimuli in seasonal affective disorder ${ }^{2}$
}

\author{
Kathryn Tierney Lindsey ${ }^{\mathrm{a}, 1}$, Kelly J. Rohan ${ }^{\mathrm{b}, *}$, Kathryn A. Roecklein ${ }^{\mathrm{c}}$, Jennifer N. Mahon ${ }^{\mathrm{b}}$ \\ a Department of Medical and Clinical Psychology, Uniformed Services University of the Health Sciences, United States \\ ${ }^{\mathrm{b}}$ Department of Psychology, University of Vermont, United States \\ c Department of Psychology, University of Pittsburgh, United States
}

\section{A R T I C L E I N F O}

\section{Article history:}

Received 24 November 2010

Received in revised form 28 February 2011

Accepted 21 April 2011

Available online 19 May 2011

\section{Keywords:}

Seasonal affective disorder

Depression

Psychophysiology

Surface facial EMG

Conditioning

\begin{abstract}
A B S T R A C T
Background: Learned associations between depressive behavior and environmental stimuli signaling low light availability and winter season may play a role in seasonal affective disorder (SAD). The purpose of this study was to determine whether light and season environmental cues elicit emotional responses that are distinct in individuals with SAD.

Methods: Twenty-four currently depressed SAD participants were compared to 24 demographicallymatched controls with no depression history on emotional responses to outdoor scenes captured under two light intensity (i.e., clear, sunny vs. overcast sky) and three season (i.e., summer with green leaves, fall with autumn foliage, and winter with bare trees) conditions. Emotion measures included surface facial electromyography (EMG) activity in the corrugator supercilii and zygomaticus major muscle regions, skin conductance, and self-reported mood state on the Profile of Mood States Depression-Dejection Subscale.

Results: Light intensity was a more salient cue than season in determining emotional reactions among SAD participants. Relative to controls, SAD participants displayed more corrugator activity, more frequent significant skin conductance responses (SCR), greater SCR magnitude, and more self-reported depressed mood in response to overcast stimuli and less corrugator activity, lower SCR magnitude, and less self-reported depressed mood in response to sunny stimuli.

Limitations: Study limitations include the single, as opposed to repeated, assessment and the lack of a nonseasonal depression group.

Conclusions: These findings suggest that extreme emotional reactivity to light-relevant stimuli may be a correlate of winter depression; and future work should examine its potential onset or maintenance significance.
\end{abstract}

(c) 2011 Elsevier B.V. All rights reserved.

\footnotetext{
is This study was supported by grants R03 MH0659 from the National Institute of Mental Health and C072EJ from the Uniformed Services University of the Health Sciences (USUHS) to Kelly J. Rohan. The opinions and assertions expressed herein are those of the authors and are not to be construed as expressing the views of the USUHS or the United States Department of Defense.

* Corresponding author at: Department of Psychology, University of Vermont, John Dewey Hall, 2 Colchester Avenue, Burlington, VT 05405-0134, United States. Tel.: + 1802656 0798; fax: +1 8026568783.

E-mail address: kelly.rohan@uvm.edu (K.J. Rohan).

${ }^{1}$ Now at Behavioral Health Department, National Naval Medical Center, Bethesda, MD, United States.
}

\section{Introduction}

Winter seasonal affective disorder (SAD) is a recurrent subtype of depression, characterized by major depression onset in the fall/winter and remission in the spring (Rosenthal et al., 1984). Etiological models propose that seasonallylinked environmental factors trigger depression onset and remission in SAD-vulnerable individuals. Most theories focus on light deprivation, specifically, as the primary environmental stressor that influences the development of depressive symptoms in SAD (Sohn and Lam, 2005). This hypothesis is 
indirectly supported by the efficacy of light therapy in acute SAD treatment (Golden et al., 2005); epidemiological research in the Northern hemisphere suggesting that SAD prevalence increases with latitude (Mersch et al., 1999); the finding that photoperiod is significantly related to weekly risk for SAD episode onset (Young et al., 1997); and negative correlations between SAD symptom severity and photoperiod, minutes of sunshine, and solar radiation (Molin et al., 1996).

Rohan et al. (2004) hypothesized that, due to the repeated pairing of low light conditions in the winter with depression in individuals with SAD, environmental cues signaling low light availability may become conditioned stimuli for eliciting negative emotional states. Similarly, because increased light availability in the spring has been repeatedly paired with amelioration of depression in SAD, environmental cues signaling sunshine may elicit a positive mood shift. Two preliminary studies support this hypothesis.

In a sample of women with SAD history; Rohan et al. (2003) found a greater exacerbation of baseline depressed mood state after exposure to photographs of outdoor scenes with an overcast sky as compared to scenes depicting a clear, sunny sky and scenes of indistinguishable light intensity (i.e., outdoor scenes that did not include the sky). In addition, women with a history of SAD reported an improvement in mood after exposure to scenes with a sunny sky relative to female controls with no history of depression. These withinand between-group differences were robust across assessments in the fall, winter, and summer seasons. Therefore, in contrast to never-depressed women who did not show differential emotional responses to stimuli varying in light conditions, women with a history of SAD exhibited a consistent pattern of emotional reactivity to light-relevant stimuli across the seasons, even during summer when SAD symptoms typically remit.

In a longitudinal comparison of college women with subsyndromal SAD (S-SAD) and nondepressed, low seasonality college women, Rohan et al. (2004) reported a similar pattern of findings in self-reported emotional responses to the same stimuli used in the 2003 study. In addition, increased psychophysiological reactivity to overcast stimuli was observed in the S-SAD group. Across winter and nonwinter seasons of assessment, women with S-SAD consistently demonstrated greater skin conductance response (SCR) magnitude in reaction to overcast scenes than controls. Women with S-SAD also exhibited higher SCR magnitude in reaction to scenes depicting overcast conditions than to scenes depicting sunny or indistinguishable light intensity conditions, regardless of season assessed. Findings from these two studies are consistent with the hypothesis that low light stimuli, even when non-threatening such as viewing a picture of an overcast sky, may be conditioned stimuli for eliciting a negative mood shift in individuals with history of winter depression.

As opposed to Rohan et al.'s (2003, 2004) manipulation of the sunny vs. overcast stimulus dimension, Sigmon et al. (2007) investigated emotional reactivity to stimuli varying in seasonal content in SAD. Individuals with SAD in a current winter depressive episode, currently depressed individuals with nonseasonal major depression, and controls with no history of depression watched two videos of outdoor scenes (one recorded in the summer and one recorded in winter). Consistent with prediction, the SAD group exhibited a higher frequency of significant skin conductance responses and greater SCR magnitude during the winter video as compared to the other two groups. The SAD and nonseasonal depression groups reported a greater exacerbation of depressed mood following the winter video as compared to controls.

Based on these studies, we propose that SAD involves learned emotional responses to environmental cues signaling low light availability (e.g., overcast skies) and the winter season (e.g., stark trees). Prior studies are limited in fully testing this hypothesis because they manipulated only one stimulus dimension (i.e., light or season cues, not both); did not control for idiosyncratic differences in scene content (i.e., the same stimulus scenes were not displayed under each condition); and restricted psychophysiological assessments to measures of skin conductance, which reflects general sympathetic arousal and, thus, tracks emotional arousal rather than emotional valence. In contrast to skin conductance, surface facial electromyography (EMG) represents a more appropriate psychophysiological measure in testing our hypothesis because it reflects emotional valence. Facial muscle group activity has been linked to specific emotions (Schwartz et al., 1976). Specifically, patterns of corrugator supercilii muscle activity (i.e., brow-pursing where the eyebrow draws downward and medialward) reliably corroborate a reported negative affective state during unpleasant or unhappy imagery; and, conversely, patterns of zygomaticus major activity (i.e., raising the corners of the mouth upwards towards the cheeks into a smile) corroborate a reported positive affective state during pleasant or happy imagery in nonclinical and depressed samples (e.g., Cacioppo et al., 1992; Greden et al., 1986). Facial muscle responses to emotional stimuli have not yet been examined in individuals with SAD. We propose that emotional responses, including facial expressions of emotion, emotional arousal, and self-reported mood state, differ according to light- and season-relevant environmental cues in SAD.

The purpose of this study was to determine whether light and season environmental cues elicit emotional responses that are distinct in individuals with SAD. To this end, currently depressed individuals with SAD and never-depressed, low seasonality controls were compared on surface facial EMG reactions in the corrugator supercilii and the zygomaticus major muscle regions, skin conductance, and self-rated mood states when exposed to digital photographs of the same series of outdoor scenes captured under two light intensity (clear, sunny vs. overcast sky) and three season (i.e., summer with green leaves, fall with autumn foliage, and winter with bare trees) conditions. Stimuli depicting fall season were included in an exploratory vane because it is possible that our hypothesis may extend to learned emotional responses to environmental cues signaling the arrival of fall, given that initial SAD symptoms typically begin in the fall. We hypothesized that the combination of light intensity and season cues would be more salient than either light or season cues alone in determining distinct emotional reactivity among SAD patients relative to controls. Specifically, we hypothesized that SAD participants would exhibit: (1) increased corrugator activity, more frequent significant skin conductance responses and greater SCR magnitude, and greater depressed mood in response to overcast/winter stimuli relative to control participants; (2) increased zygomaticus activity and improved mood in response 
to sunny/summer stimuli relative to controls; (3) increased corrugator activity, more frequent significant skin conductance responses and greater SCR magnitude, and greater depressed mood in response to overcast/winter stimuli relative to sunny/ summer stimuli; and (4) increased zygomaticus activity and improved mood in response to sunny/summer stimuli relative to overcast/winter stimuli.

Although there are six light intensity/season conditions in this study (i.e., overcast/summer, overcast/fall, overcast/winter, sunny/summer, sunny/fall, sunny/winter), our a priori hypotheses focused on the two (i.e., overcast/winter vs. sunny/summer) where the most extreme between-group differences and withinSAD group differences were expected. However, the remaining four conditions complete the picture in providing information about which stimulus dimension(s) are the most salient in determining emotional reactions in SAD: (a) the hypothesized interaction of light and season cues, (b) light cues alone, or (c) season cues alone. In the latter two cases, the groups would not differ within any individual light intensity/season condition (as we hypothesized); but the groups would generally differ on either the light intensity or season stimulus dimension, regardless of which cues from the other dimension were represented. In the case that light or season was the salient dimension as opposed to their combination, the SAD group would also not show any differences between individual light intensity/season conditions (as we hypothesized), but would show general differences between the sunny vs. overcast light intensities or between the depicted seasons, regardless of which cues from the other dimension were present.

\section{Method}

\subsection{Participant screening and inclusion criteria}

Adult male and female participants, aged 18 or older, were recruited through media advertisements in the greater Washington, DC metro area. Inclusion criteria for the SAD group included a diagnosis of Major Depression, Recurrent, with a Seasonal Pattern on the Structured Clinical Interview for DSM-IV Axis I Disorders (SCID; First et al., 1995) and meeting Structured Interview Guide for the Hamilton Rating Scale for Depression-Seasonal Affective Disorder Version (SIGH-SAD; Williams et al., 1992) criteria for a current SAD episode (i.e., total SIGH-SAD score $\geq 20+21$-item Structured Interview Guide for Hamilton Rating Scale for Depression score $\geq 10$ + atypical subscale score $\geq 5$; Terman et al., 1990). Exclusion criteria included any comorbid Axis I Disorder and current treatment (e.g., light therapy, psychotherapy, or psychotropic medications). Inclusion criteria for control participants were: no current Axis I psychopathology and no history of past major depressive episodes on the SCID, a Seasonal Pattern Assessment Questionnaire (SPAQ; Rosenthal et al., 1987) Global Seasonality Score (GSS) in the normal range [i.e., either (a) GSS $=8$ or 9 and endorsing no problems across the seasons or (b) GSS $\leq 7$ ], and a normal range score of $\leq 13$ on the Beck Depression Inventory-Second Edition (BDI-II; Beck et al., 1996). A licensed psychologist with substantial experience in diagnosing and treating SAD and depression (KJR) administered the SCID to 16 (33\%) of the participants who qualified for the study. An advanced clinical graduate student with SCID training and experience admin- istered the SCID to remaining 32 (67\%) qualified participants with the diagnosis subsequently verified by the licensed psychologist's review of an audiotape of the SCID prior to inclusion. During recruitment, efforts were made to match the SAD and control groups on age, sex, and race.

\subsection{Stimuli development: pilot studies}

To develop the stimuli, we digitally photographed potential stimulus scenes, all depicting deciduous trees, grass, and sky at local parks in the summertime. The initial pool of 24 digital photographs consisted of 12 pairs with each pair including the same outdoor scene photographed once under a sunny, clear sky and again under overcast conditions. We conducted a pilot study on a convenience sample of 24 university students and faculty members to identify any scenes that were intrinsically negative or positive, regardless of light intensity. We selected the five pairs of scenes from the original 12 pairs that showed the highest positive correlations between Likert ratings on a 7-point scale for elicited emotion ( 1 = "very sad" and $7=$ "very happy") and light intensity ( 1 = "dark/dreary" and $7=$ "bright/sunny") as the most appropriate stimulus pairs. The five selected scenes were subsequently photographed during the other seasons (i.e., in the fall as the leaves were changing colors and in the winter with bare trees) and under both light intensity conditions. Each time, efforts were made to capture each stimulus scene from the same spot and angle. This was accomplished by on-thespot comparisons to the prior photographs of each stimulus scene such that, to the naked eye, the elements of the scene composition appeared nearly identical in size and position across all six photographs. See Fig. 1 for an example of a stimulus scene under each light intensity/season condition.

To validate the selected stimuli, we conducted a pilot study using 15 currently depressed SAD patients in the winter. Participants viewed each of the five stimulus scenes as photographed under all six light intensity/season conditions: overcast/summer, overcast/fall, overcast/winter, sunny/summer, sunny/fall, and sunny/winter. In general, the SAD sample reported mood states on the Profile of Mood States Depression-Dejection Subscale (POMS; McNair et al., 1971) that fluctuated in the predicted directions by stimulus type. Median mood ratings indicated improved mood immediately after viewing blocks of sunny/summer stimuli and exacerbated depressed mood subsequent to viewing blocks of overcast/winter stimuli.

\subsection{Psychophysiological task}

The final set of stimuli consisted of 30 digital photographs with each of the five scenes represented under six conditions in a 2 (light intensity; clear and sunny sky, overcast sky) $\times 3$ (seasonal cues; summer with green leaves, fall with autumn foliage, and winter with bare trees) repeated measures design. The task procedures involved a 2-minute adaptation and a 3-minute baseline followed by presentation of the six stimulus blocks. The stimulus block presentation order was counterbalanced across groups. Each scene was displayed for $10 \mathrm{~s}$ on a PC monitor followed by a 10 -s poststimulus interval where nothing was presented. Participants were instructed to imagine what they would be thinking and 

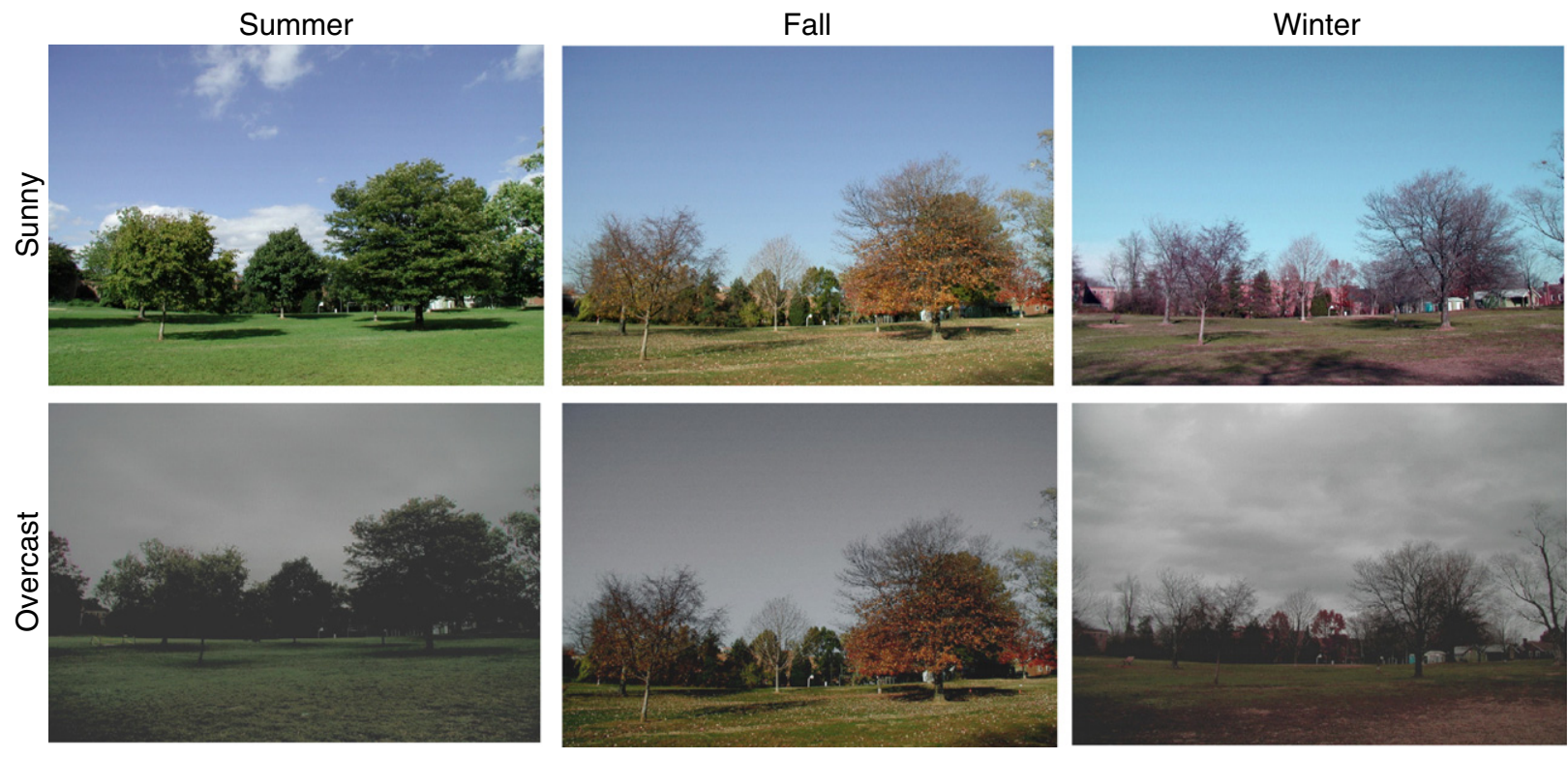

Fig. 1. Example of a stimulus scene captured under each of the two light intensity (overcast, sunny) and each of the three season (summer, fall, winter) conditions.

feeling if they were in the scene. Participants completed the POMS Depression-Dejection Subscale after baseline and after each stimulus block.

Surface facial EMG activity in the corrugator and zygomaticus regions and electrodermal activity were recorded using PSYLAB software (Dow, 1991) operated on Contact Precision Instruments psychophysiological equipment (London, United Kingdom). After cleansing and lightly abrading the skin, three pairs of $0.40-\mathrm{cm} \mathrm{Ag}-\mathrm{AgCl}$ electrodes were placed on the corrugator muscle, the upper zygomaticus, and the lower zygomaticus. Prior to recording, the EMG signal was calibrated via an internal calibration signal. Impedance levels of $<10 \mathrm{k} \Omega$ were considered acceptable. The EMG passband for analysis was $80-250 \mathrm{~Hz}$, and the filter settings included a low pass filter (LPF) setting of $500 \mathrm{~Hz}$ and a high pass filter (HPF) setting of $30 \mathrm{~Hz}$. The sampling rate was $1000 \mathrm{~Hz}$ as per the Nyquist sampling theorem (Cutmore and James, 1999). The signal was first recorded as a raw EMG signal. The system automatically converted the continuous analog signal into a digital signal and rectified and smoothed the data.

In this study, there were three measures of electrodermal activity: skin conductance level (SCL), the significant skin conductance response (SCR), defined as a change of at least $0.05 \mu \mathrm{S}$ from baseline SCL summed within type of stimulus (Venables and Christie, 1980), and SCR magnitude (i.e., the magnitude of the SCR with the largest response; Venables and Christie, 1980). Skin conductance was measured directly using DC coupling with constant voltage electrode excitation (Dow, 1991) via electrodes placed in a bipolar arrangement involving the medial phalanx of the third and fourth fingers on the participants' non-dominant hand. Prior to measurement, automatic calibration took place switching from $0 \mu \mathrm{S}$ (open circuit) to $100 \mu \mathrm{S}(0.1 \% 10 \mathrm{k} \Omega$ precision resistance). A $10 \mathrm{~Hz}$ filter was applied to the SCR response signal to prevent aliasing. The pre-amplifier power box of the Precision Instruments equipment (London, United Kingdom) sample rate was set at $40 \mathrm{~Hz}$ (Dow, 1991). The high resolution of the system allowed SCRs below $0.01 \mu \mathrm{S}$ to be amplified, rectified, and smoothed via the software.

\subsection{Profile of Mood States (POMS)}

The Profile of Mood States Depression-Dejection Subscale (POMS; McNair et al., 1971), a state measure of transient selfreported depressed mood is a 15 -item subscale from the original 65-item measure. Respondents indicate the degree to which they are currently feeling ("at that moment") each emotion (e.g., sad, unworthy, hopeless) on a 5-point Likert scale ranging from 0 ("not at all") to 4 ("extremely"). The POMS Depression-Dejection Subscale has good psychometric properties, including an internal consistency of 0.95 and a strong positive correlation with measures of depressive symptomatology (McNair et al., 1971). In this study, Cronbach's $\alpha$ for the POMS-D at baseline $=0.91$.

\subsection{Data analysis}

Psychophysiological dependent measures were collected during the 10-s interval of stimulus presentation and included mean EMG in each of three muscle regions (i.e., the corrugator supercilii and the lower and upper zygomaticus major) and three skin conductance variables [i.e., skin conductance level (SCL), significant skin conductance responses (SCRs), and SCR magnitude]. In accordance with the recommendations of Bush et al. (1993), each of the psychophysiological measures was standardized to reduce variability across participants. The transformation consisted of (Bush et al., 1993): (1) generating the mean and SD of all the scores for each participant across the 30 slides in all light/season experimental conditions, (2) transforming each participant's 30 raw scores into $\mathrm{Z}$ scores by subtracting his/her mean and dividing by his/her SD, and (3) computing the mean of each 


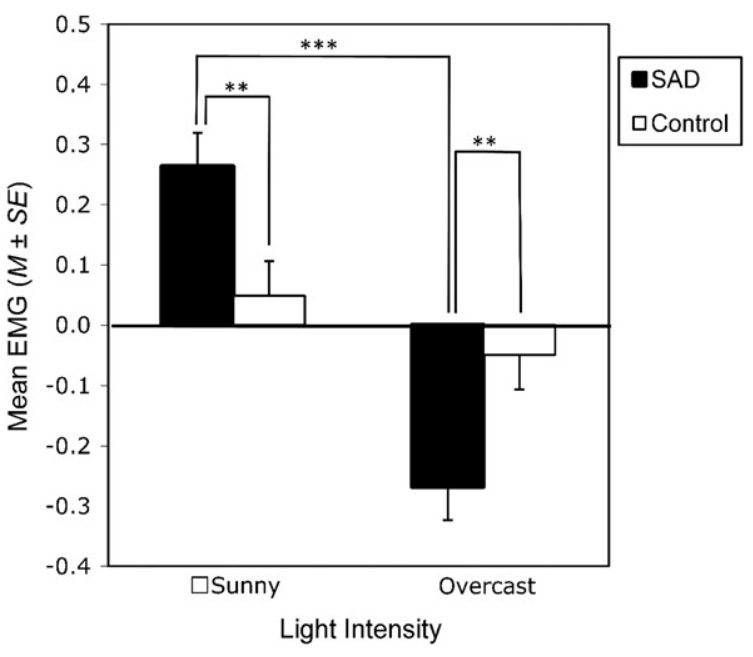

Fig. 2. Mean EMG corrugator supercilii activity during stimulus presentation, collapsing across seasons. Values are based on the Z-score transformation described in the Method section. Negative values indicate greater corrugator supercilii activity relative to baseline (i.e., a more negative emotional reaction in the form of more brow pursing). Positive values indicate less corrugator supercilii activity relative to baseline (i.e., a more positive emotional reaction in the form of less brow pursing). ${ }^{* *} p<0.001$.

participant's Z scores within each of the six light/season conditions, yielding a single number for each condition as the unit of analysis. In addition, the above transformations were conducted using change scores from the 3-minute baseline period (i.e., the mean of the 1810 -s baseline epochs).

To examine shifts in self-reported mood to the different types of stimuli, we calculated POMS Depression-Dejection change scores by subtracting each post-stimulus block POMS from the baseline POMS, as in our prior studies (Rohan et al.,
2003, 2004). Negative change scores indicate an exacerbation of mood and positive change scores indicate an improvement in mood relative to baseline (Fig. 3).

We conducted a series of 2 (group; SAD, control) $\times 2$ (light intensity; sunny, overcast) $\times 3$ (season; summer, fall, winter) mixed-design ANOVAs on the above dependent measures with group as the between-subjects factor and with light intensity and season as within-subjects factors. For the hypothesized pattern of findings, a significant Group $\times$ Light intensity $\times$ Season interaction was predicted for each analysis. If a significant 3-way interaction had been found, the plan was to follow it up with the tests of 2-way interaction effects examining the Group $\times$ Light interaction within each level of season. If a 2-way interaction within a season was found, simple main effect analyses would have been performed followed by post hoc contrasts. However, results did not reveal any 3-way interactions. Instead, significant overall 2-way interactions were a common finding and were followed up with simple main effect analyses and then, for any season differences within group or light intensity, with Tukey's HSD pairwise comparisons.

\section{Results}

\subsection{Participant characteristics}

Sample demographic characteristics are presented in Table 1 . The SAD $(N=24)$ and control $(N=24)$ groups did not differ significantly on age, sex, race, marital status, highest education level, or employment status, all ps ns. As expected, SAD participants $(M=28.33 \pm 4.93$ and $26.17 \pm 7.11)$ endorsed significantly greater current depression severity than controls $(M=2.63 \pm 2.79$ and $1.75 \pm 2.45)$ on the SIGH-SAD and BDI-II, respectively. The groups did not differ on two potential confounding variables we examined in secondary analyses,
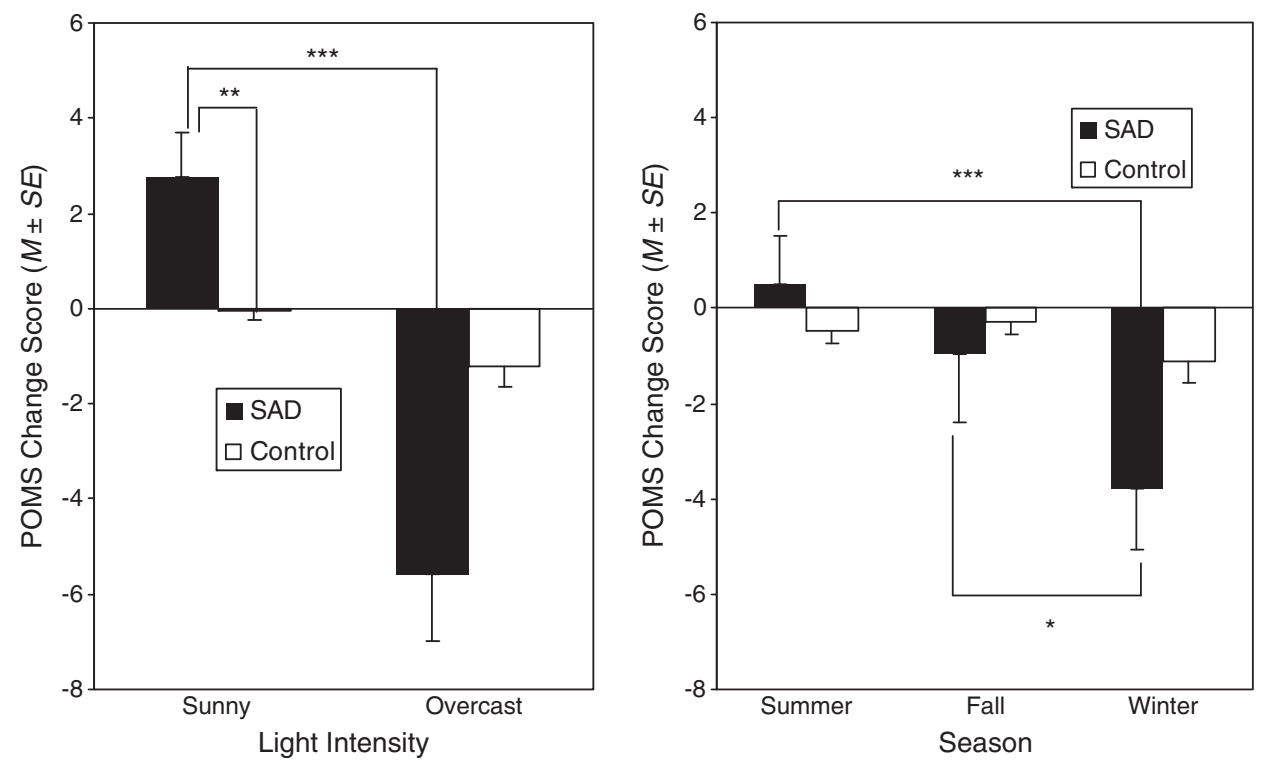

Fig. 3. Profile of Mood States Depression-Dejection Subscale change scores from baseline, collapsing across seasons (left) and collapsing across light intensities (right). Change score = baseline POMS - post-stimulus block POMS. Negative change scores indicate an exacerbation of mood and positive change scores indicate an improvement in mood relative to baseline. ${ }^{* *} p<0.001,{ }^{* *} p<0.01$, and ${ }^{*} p<0.05$. 
Table 1

Sample demographic characteristics.

\begin{tabular}{|c|c|c|c|}
\hline & $\begin{array}{l}\text { SAD } \\
(n=24)\end{array}$ & $\begin{array}{l}\text { Control } \\
(n=24)\end{array}$ & Statistic \\
\hline Age (years), $M(\mathrm{SD})$ & $41.58(11.72)$ & $41.83(12.75)$ & $\begin{array}{l}t(46)=-0.07 \\
p=0.94\end{array}$ \\
\hline \multicolumn{4}{|l|}{ Sex, $n(\%)$} \\
\hline Female & $22(92)$ & $22(92)$ & $X^{2}(1, N=48)=$ \\
\hline Male & $2(8)$ & $2(8)$ & $1.00, p=1.00$ \\
\hline \multicolumn{4}{|l|}{ Race, $n(\%)$} \\
\hline White & $17(71)$ & $17(71)$ & $X^{2}(1, N=48)=$ \\
\hline Minority & 7 (29) & 7 (29) & $1.00, p=1.00$ \\
\hline Asian & $2(8)$ & $0(0)$ & \\
\hline African American & $4(17)$ & $4(17)$ & \\
\hline Hispanic & $0(0)$ & $2(8)$ & \\
\hline Other & $1(4)$ & $1(4)$ & \\
\hline \multicolumn{4}{|l|}{ Marital status, $n(\%)$} \\
\hline Single & $8(33)$ & 7 (29) & $X^{2}(3, N=48)=$ \\
\hline Married & $11(46)$ & $16(67)$ & $4.99, p=0.17$ \\
\hline Living together & 4 (17) & $0(0)$ & \\
\hline Divorced & $1(4)$ & $1(4)$ & \\
\hline \multicolumn{4}{|l|}{$\begin{array}{l}\text { Highest education level, } \\
n(\%)\end{array}$} \\
\hline High school diploma & $6(25)$ & $6(25)$ & $X^{2}(2, N=48)=$ \\
\hline Undergraduate degree & $11(46)$ & $12(50)$ & $0.12, p=0.94$ \\
\hline Graduate degree & 7 (29) & $6(25)$ & \\
\hline \multicolumn{4}{|l|}{ Employment status, $n$ (\%) } \\
\hline Employed & $23(96)$ & $22(92)$ & $X^{2}(2, N=48)=$ \\
\hline Homemaker & $1(4)$ & $1(4)$ & $1.02, p=0.60$ \\
\hline Retired & $0(0)$ & $1(4)$ & \\
\hline
\end{tabular}

including imagery ability on the Vividness of Visual Imagery Questionnaire (VVIQ; White et al., 1977) and the proportion of females in the premenstrual (late luteal) phase of the menstrual cycle based on self-report ( $n=2$ in each group).

\subsection{Results overview}

Table 2 presents a summary of the significant betweengroup, within-group, and overall between-stimulus differences for each outcome measure.

\subsection{Surface facial EMG responses}

\subsubsection{Corrugator supercilii}

An ANOVA on mean EMG corrugator activity revealed a significant Group $\times$ Light interaction, Wilks' lambda $F(1,46)=$ 7.33, $p=0.009, \eta^{2}=0.137$, and a significant Light $\times$ Season interaction, Wilks' lambda $F(2,45)=3.93, p=0.027, \eta^{2}=$ 0.149 . Main effect analyses revealed a group main effect for both the overcast, $F(1,46)=7.44, p=0.009, \eta^{2}=0.139$, and sunny, $F(1,46)=7.22, p=0.010, \eta^{2}=0.136$, light intensity conditions, collapsing across seasons. Specifically, the SAD group had significantly greater corrugator activity during overcast slides and less corrugator activity during sunny slides than controls. Main effect analyses further revealed a main effect of light, collapsing across seasons, within the SAD group, $F(1,46)=21.94, p<0.001, \eta^{2}=0.323$, whereby SAD participants showed increased corrugator activity during exposure to overcast as compared to sunny stimuli (see Fig. 2).

In follow up of the Light $\times$ Season interaction, main effect analyses revealed a main effect of light for summer stimuli, collapsing across groups, $F(1,46)=18.22, p<0.001$, $\eta^{2}=0.284$, indicating greater corrugator activity during overcast/summer than sunny/summer slides. A main effect of season for sunny stimuli, collapsing across groups, was also found, Wilks' lambda $F(1,46)=12.90, p<0.001, \eta^{2}=0.364$. Post hoc comparisons revealed greater mean EMG corrugator in response to sunny/winter than sunny/summer $(p<0.001$, $d=0.37$ ) and to sunny/fall than sunny/summer slides $(p=0.002, d=0.77)$.

\subsubsection{Zygomaticus major}

For the lower zygomaticus, the ANOVA revealed an overall light main effect, Wilks' lambda $F(1,46)=11.63, p=0.001$, $\eta^{2}=0.202$, indicating greater zygomaticus activity during sunny than overcast slides. An overall season main effect was also found, Wilks' lambda $F(2,45)=3.64, p=0.034, \eta^{2}=0.139$, with greater mean EMG to summer than to winter $(p=0.017$, $d=0.64)$ or fall ( $p=0.026, d=0.55)$ slides. For the upper zygomaticus, there was an overall light main effect, Wilks' lambda $F(1,46)=12.50, p=0.001, \eta^{2}=0.214$, with greater activity in the upper zygomaticus during sunny than overcast slides.

\subsection{Skin conductance}

For significant skin conductance responses (SCRs), the ANOVA revealed a Group $\times$ Light interaction, Wilks' lambda $F(1,46)=4.07, p=0.050, \eta^{2}=0.081$, and a Light $\times$ Season interaction, Wilks' lambda $F(2,45)=3.51, p=0.038, \eta^{2}=$ 0.135 . Main effect analyses revealed a group main effect for overcast slides, $F(1,46)=4.15, p=0.047, \eta^{2}=0.083$, whereby the SAD group had more frequent SCRs than

Table 2

Summary of significant findings.

\begin{tabular}{|c|c|c|c|}
\hline Outcome measure & Between-group differences & Within-group differences & Overall between-stimulus differences \\
\hline Mean EMG corrugator supercilii & $\begin{array}{l}\mathrm{SAD}>\text { controls to overcast } \\
\mathrm{SAD}<\text { controls to sunny }\end{array}$ & SAD: overcast $>$ sunny & $\begin{array}{l}\text { Overcast/summer }>\text { sunny/summer } \\
\text { Sunny/winter }>\text { sunny/summer } \\
\text { Sunny/fall }>\text { sunny/summer }\end{array}$ \\
\hline Mean EMG lower zygomaticus major & & & $\begin{array}{l}\text { Sunny }>\text { overcast } \\
\text { Summer }>\text { winter }\end{array}$ \\
\hline Mean EMG upper zygomaticus major & & & Sunny $>$ overcast \\
\hline Significant skin conductance responses & SAD $>$ controls to overcast & & Sunny/fall $<$ sunny/summer \\
\hline Skin conductance response magnitude & $\begin{array}{l}\mathrm{SAD}>\text { controls to overcast } \\
\mathrm{SAD}<\text { controls to sunny }\end{array}$ & & $\begin{array}{l}\text { Fall }>\text { summer } \\
\text { Fall }>\text { winter }\end{array}$ \\
\hline $\begin{array}{l}\text { Profile of Mood States Depression-Dejection } \\
\text { change scores }\end{array}$ & $\begin{array}{l}\text { SAD }>\text { controls to overcast } \\
\text { SAD }<\text { controls to sunny }\end{array}$ & $\begin{array}{l}\text { SAD: overcast }>\text { sunny } \\
\text { SAD: winter }>\text { summer } \\
\text { SAD: winter }>\text { fall }\end{array}$ & \\
\hline
\end{tabular}


controls during overcast slides. Main effect analyses also found a main effect of season for sunny stimuli, Wilks' lambda $F(2,45)=3.21, p=0.050, \eta^{2}=0.125$, with more SCRs during sunny/fall than sunny/summer slides $(p=0.023, d=0.51)$.

For SCR magnitude, the ANOVA revealed a Group $\times$ Light interaction, Wilks' lambda $F(1,46)=4.20, p=0.046$, $\eta^{2}=0.084$, and an overall season main effect, Wilks' lambda $F(2,45)=3.93, p=0.026, \eta^{2}=0.149$, with greater SCR magnitude during fall than summer $(p=0.020, d=0.77)$ or winter $(p=0.035, d=0.48)$ slides. Main effect analyses revealed a group main effect for both sunny, $F(1,46)=4.15$, $p=0.047, \eta^{2}=0.083$, and for overcast stimuli, $F(1,46)=$ 4.26, $p=0.045, \eta^{2}=0.084$. Specifically, the SAD group had greater SCR magnitude during overcast stimuli and lower SCR magnitude during sunny stimuli than controls. No significant interactions or main effects were revealed for skin conductance level (SCL).

\subsection{Depressed mood ratings}

The ANOVA on POMS Depression-Dejection Subscale change scores from baseline revealed a significant Group $\times$ Light interaction, Wilks' lambda $F(1,46)=29.75, p<0.001$, $\eta^{2}=0.393$, and a significant Group $\times$ Season interaction, Wilks' lambda $F(2,45)=4.98, p=0.011, \eta^{2}=0.181$. In follow up of the Group $\times$ Light interaction, main effect analyses revealed a main effect of group, collapsing across seasons, within the sunny, Wilks' lambda $F(1,46)=8.92$, $p=0.005, \eta^{2}=0.162$, and within the overcast stimuli, Wilks' lambda $F(1,46)=8.90, p=0.005, \eta^{2}=0.162$. Specifically, SAD participants reported a greater exacerbation of depressed mood subsequent to overcast stimuli $(M=-5.60)$ as compared to controls $(M=-1.21)$; and SAD participants $(M=2.76)$ reported a greater improvement in depressed mood after viewing sunny stimuli relative to controls $(M=$ -0.04 ). Main effect analyses further revealed a main effect of light, collapsing across seasons, within the SAD group, Wilks' lambda $F(1,46)=80.36, p<0.001, \eta^{2}=0.636$, whereby SAD participants reported greater exacerbation of baseline depressed mood after viewing overcast stimuli than sunny stimuli.

In follow up of the Group $\times$ Season interaction, main effect analyses revealed a main effect of season, collapsing across light intensities, within the SAD group, Wilks' lambda $F(2,45)=14.12, p<0.001, \eta^{2}=0.386$. SAD participants reported greater exacerbation of depressed mood after viewing winter stimuli $(M=-3.77)$ as compared to summer $(M=0.50), t(23)=-3.83, p=0.001, d=0.76$, and fall stimuli $(M=-0.98), t(23)=-2.10, p=0.047, d=0.91$. Main effect analyses revealed no group main effect for winter, summer, or fall season stimuli, collapsing across light intensities.

\section{Discussion}

This study investigated whether currently depressed individuals with SAD demonstrate differential emotional responses, including facial expressions of emotion, emotional arousal, and self-rated mood state, when exposed to visual stimuli that vary in light intensity and seasonal cues relative to demographically-matched, never-depressed controls. This is the first study to examine both light- and season-relevant environmental cues to explicate the specific stimulus dimension(s) eliciting emotional responses in SAD, thereby identifying the possible types of conditioned stimuli eliciting depressive behavior in SAD. Light intensity was varied by manipulating whether the sky depicted sunny or overcast weather conditions. Season was varied by manipulating the appearance of the trees in summer with green leaves, in fall with autumn foliage, and in winter with bare branches. In an improvement over prior methods, stimuli were composed of the same series of outdoor scenes photographed under each of the six light intensity and season conditions: overcast/summer, overcast/fall, overcast/winter, sunny/summer, sunny/fall, and sunny/winter. It was hypothesized that SAD patients would have distinct emotional responses to these stimuli relative to controls and that the interaction of light intensity and season cues would be more salient than either light or season cues alone in determining emotional responses among SAD patients.

In contrast to the proposed interaction, light intensity appeared to be more salient than seasonal cues in determining facial expressions of emotion that were distinct in SAD patients. For mean EMG in the corrugator supercilii muscle, SAD participants showed greater activity (i.e., more browpursing or frowning) in response to overcast slides and less activity (i.e., less brow-pursing) in response to sunny slides as compared to controls. SAD participants also showed greater corrugator activity during overcast than sunny slides, whereas controls did not differ on their corrugator responses to the overcast vs. sunny slides. This indicates an extreme emotional response, in terms of facial muscle patterning in the corrugator, to outdoor visual stimuli that vary in light intensity among SAD patients. In other words, individuals with SAD may be unique in contracting their corrugator muscle to a greater extent than "normal" in reaction to overcast stimulus and to a lesser extent than "normal" in reaction to a sunny stimulus. This indicates both a more negative emotional reaction to overcast stimuli and a less negative emotional reaction to sunny stimuli in SAD patients, specifically.

Light and season cues had interactive effects in determining facial expressions of emotion in the corrugator across the SAD and control groups. Participants, in general, showed greater corrugator activity (i.e., more brow-pursing) during overcast/summer than sunny/summer slides and during sunny/winter and sunny/fall than sunny/summer slides. This suggests that people, regardless of SAD status, show more negative facial expressions in the corrugator to: (a) overcast than to sunny conditions when summer-relevant cues are present and (b) winter and fall relative to summer cues when sunny conditions are depicted. Therefore, people, in general, may have a hyperpositive corrugator response to sunny/summer stimuli relative to any other combination of light intensity and season cues.

Corrugator responses to environmental stimuli appear to be more SAD-specific than zygomaticus major responses as only corrugator responses distinguished between the patient and control groups in facial expressions of emotion during the stimuli. Instead, light intensity and season had independent influences on facial expressions in the zygomaticus region across participants, in general. In both the upper and lower zygomaticus, greater activity (i.e., more smiling) was observed during sunny as compared to overcast stimuli. In the 
lower zygomaticus, greater activity was observed during summer than winter or fall slides. This general pattern of results reflects that people with or without SAD smile more to sunny than to overcast conditions, regardless of season cues present, and in reaction to summer than to fall or winter season cues, regardless of whether it is sunny or overcast.

This study incorporated psychophysiological measures of both emotional valence (i.e., surface facial EMG) and emotional arousal (i.e., skin conductance). Skin conductance results are generally consistent with the facial EMG results for the corrugator as they indicate that SAD participants had more frequent significant skin conductance responses and greater SCR magnitude when viewing overcast stimuli and lower SCR magnitude when viewing sunny stimuli than controls. Therefore, in terms of corrugator and skin conductance reactions, SAD patients showed a unique negative emotional response to overcast stimuli and a unique positive emotional response to sunny stimuli, which was not observed in controls. In contrast, there were some interactive and solo effects of season on the emotional arousal in participants, in general, with fall cues eliciting the most negative responses (i.e., more frequent significant skin conductance responses during sunny/fall than sunny/summer slides and greater SCR magnitude during fall than summer or winter slides).

Light intensity and seasonal cues appear to be distinct and salient determinants of self-reported mood state unique to SAD patients. Relative to controls, SAD participants reported improved mood following blocks of sunny stimuli (regardless of season) and an exacerbation of baseline depressed mood following blocks of overcast stimuli (regardless of season). SAD participants also reported an exacerbation of baseline depressed mood following overcast stimuli (regardless of season) as compared to sunny stimuli and following winter stimuli (regardless of light intensity) as compared to summer and fall stimuli.

It will be important for future studies to determine if this pattern of extreme emotional reactivity to light-relevant cues is unique to SAD or generalizes to nonseasonal depression. In a prior study, individuals with SAD exhibited a higher frequency of significant skin conductance responses and greater SCR magnitude while viewing a winter video as compared to never-depressed control and nonseasonal depression groups (Sigmon et al., 2007). However, the SAD and nonseasonal depression groups in that study both reported a greater exacerbation of depressed mood following the winter video as compared to controls. It is not known whether the lightrelated dimension of our stimuli discriminates between seasonal and nonseasonal depression groups and between nonseasonal depression and control groups. Future group comparisons using these stimuli could also longitudinally assess participants with seasonal and nonseasonal depression during a major depressive episode and during remission to determine whether any distinct patterns of emotional responding that are found in either or both groups wax and wane with depression or are more trait-like in nature. Future studies should also consider including a self-report scale that taps another emotion, particularly one reflecting positive affect and consistent with zygomaticus activity (e.g., POMS vigor), to examine the discriminant validity of self-report findings. Although surface facial EMG has been used in recent emotion research using nonclinical samples, it has not been commonly used in research with clinically depressed individuals for over two decades. The current study's design and results illustrate the potential value of including surface facial EMG as a measure of emotional valence in clinical populations, particularly in paradigms that are interested in emotional reactions to affect-laden stimuli.

In summary, light intensity was a more salient cue than season in determining emotional reactions that were unique among SAD participants. Relative to controls, SAD participants had more corrugator activity, more frequent significant skin conductance responses (SCR), greater SCR magnitude, and more self-reported depressed mood in response to overcast stimuli and less corrugator activity, lower SCR magnitude, and less self-reported depressed mood in response to sunny stimuli. Given that self-reported affect and psychophysiological responses reflect different channels of emotional responding, it is noteworthy that results converge for SAD vs. control group differences across self-reported depressed mood, EMG in the corrugator, significant skin conductance responses, and SCR magnitude. Based on this convergence of findings, we conclude that extreme emotional reactivity to light-relevant stimuli is a correlate of winter depression. Although individuals with or without SAD, in general, showed some reactivity to these types of stimuli, what was unique about SAD patients compared to neverdepressed controls was their differential reactivity to the sunny vs. overcast stimulus dimension.

Although the observed pattern of results is consistent with the hypothesis that SAD involves unique learned emotional responses to environmental cues signaling low light availability (but not cues to signaling the winter season), this study cannot distinguish between the relative contributions of classical conditioning, operant conditioning, and/or cognitive processes as mechanisms. Future studies are needed to understand how the observed associations between light-relevant stimuli and emotional responses develop, whether they have onset or maintenance significance, and their implications for SAD assessment and treatment. If these responses are related to depression recurrence in SAD, exposure-based interventions, mindfulness, and cognitive restructuring may be indicated to break associations between environmental cues related to light intensity and emotional responses in SAD. Cognitive restructuring of negative thoughts, including thoughts related to light availability, and scheduling pleasant activities to counteract depressive behavior in dreary weather are included in our cognitive-behavioral therapy for SAD treatment, which has shown efficacy in acute SAD treatment (Rohan et al., 2007) and may reduce the risk of SAD episode recurrence in the subsequent winter season (Rohan et al., 2009).

\section{Role of the funding source}

This study was supported by grants R03 MH0659 from the National Institute of Mental Health and C072EJ from the Uniformed Services University of the Health Sciences (USUHS) to Kelly J. Rohan. The NIMH and USUHS NIMH had no further role in study design; in the collection, analysis, and interpretation of data; in the writing of the report; and in the decision to submit the paper for publication. The opinions and assertions expressed herein are those of the authors and are not to be construed as expressing the views of the USUHS or the United States Department of Defense.

\section{Conflict of interest}

All authors declare that they have no conflicts of interest. 


\section{Acknowledgments}

We thank Michael Feuerstein, Willem Kop, and Mark Rollag for their comments on an earlier version of this manuscript. This work is based on the doctoral dissertation of the first author. This work was presented, in part, at the annual meeting of the Association for Behavioral and Cognitive Therapies, November 2005, Washington, DC.

\section{References}

Beck, A.T., Steer, R.A., Brown, G.K., 1996. Beck Depression Inventory-2nd Edition Manual. The Psychological Corporation, San Antonio, TX.

Bush, L.K., Hess, U., Wolford, G., 1993. Transformations for within-subjects designs: a Monte Carlo investigation. Psychological Bulletin 113, 566-579.

Cacioppo, J.T., Bush, L.K., Tassinary, L.G., 1992. Microexpressive facial actions as a function of affective stimuli: replication and extension. Personality and Social Psychology Bulletin 18, 515-526.

Cutmore, T.R.H., James, D.A., 1999. Identifying and reducing noise in psychophysiological recordings. International Journal of Psychophysiology 32, 129-150.

Dow, R., 1991. Psylab (Version 2.0). [Computer Software]. Contact Precision Instruments, London UK.

First, M.B., Spitzer, R.L., Gibbon, M., Williams, J.B., 1995. Structured Clinical Interview for DSM-IV Axis I Disorders (SCID-Clinician Version). New York State Psychiatric Institute Biometrics Research Department, New York.

Golden, R.N., Gaynes, B.N., Ekstrom, R.D., Hamer, R.M., Jacobsen, F.M., Suppes, P., Wisner, K.L., Nemeroff, C.B., 2005. The efficacy of light therapy in the treatment of mood disorders: a meta-analysis of the evidence. The American Journal of Psychiatry 162, 656-662.

Greden, J.F., Genero, N., Price, H.L., Feinberg, M., Levine, S., 1986. Facial electromyography in depression. Archives of General Psychiatry 43, 269-274.

McNair, D.M., Lorr, M., Droppleman, L.F., 1971. EITS Manual for the Profile of Mood States. Educational and Industrial Testing Service, San Diego.

Mersch, P.A., Middendorp, H.M., Bouhuys, A.L., Beersma, D.G.M., Van-denHoofdakker, R.H., 1999. Seasonal affective disorder and latitude: a review of the literature. Journal of Affective Disorders 53, 35-48.

Molin, J., Mellerup, E., Bolwig, T., Scheike, T., Dam, H., 1996. The influence of climate on development of winter depression. Journal of Affective Disorders 37, 151-155.

Rohan, K.J., Sigmon, S.T., Dorhofer, D.M., 2003. Cognitive-behavioral factors in seasonal affective disorder. Journal of Consulting and Clinical Psychology 71, 22-30.
Rohan, K.J., Sigmon, S.T., Dorhofer, D.M., Boulard, N.E., 2004. Cognitive and psychophysiological correlates of subsyndromal seasonal affective disorder. Cognitive Therapy and Research 28, 39-56.

Rohan, K.J., Roecklein, K.A., Tierney Lindsey, K., Johnson, L.G., Lippy, R.D., Lacy, T.M., Barton, F.B., 2007. A randomized controlled trial of cognitivebehavioral therapy, light therapy, and their combination for seasonal affective disorder. Journal of Consulting and Clinical Psychology 75, 489-500.

Rohan, K.J., Roecklein, K.A., Lacy, T.M., Vacek, P.M., 2009. Winter depression recurrence one year after cognitive-behavioral therapy, light therapy, or combination treatment. Behavior Therapy 40, 225-238.

Rosenthal, N.E., Sack, D.A., Gillin, J.C., Lewy, A.J., Goodwin, F.K., Davenport, Y., Mueller, P.S., Newsome, D.A., Wehr, T.A., 1984. Seasonal affective disorder: a description of the syndrome and preliminary findings with light therapy. Archives of General Psychiatry 41, 72-80.

Rosenthal, N.E., Bradt, G.H., Wehr, T.A., 1987. Seasonal Pattern Assessment Questionnaire. National Institute of Mental Health, Bethesda, MD.

Schwartz, G.E., Fair, P.L., Salt, P., Mandel, M.R., Klerman, G.L., 1976. Facial expression and imagery in depression: an electromyographic study. Psychosomatic Medicine 38 (5), 337-347.

Sigmon, S.T., Whitcomb-Smith, S., Boulard, N.E., Pells, J.P., Hermann, B.A., Edenfield, T.M., LaMattina, S.M., Shartell, J.G., 2007. Attentional bias and psychophysiological arousal in seasonal and nonseasonal depression. Cognitive Therapy and Research 31, 619-638.

Sohn, C.H., Lam, R.W., 2005. Update on the biology of seasonal affective disorder. CNS Spectrums 10, 635-646.

Terman, M., Terman, J.S., Rafferty, B., 1990. Experimental design and measures of success in the treatment of winter depression by bright light. Psychopharmacology Bulletin 26, 505-510.

Venables, P.H., Christie, M.J., 1980. Electrodermal activity. In: Martin, I., Venables, P.H. (Eds.), Techniques in Psychophysiology. John Wiley \& Sons, New York, pp. 3-67.

White, K., Sheehan, P.W., Ashton, R., 1977. Imagery assessment: A survey of self-report measures. Journal of Mental Imagery 1, 145-170.

Williams, J.B., Link, M.J., Rosenthal, N.E., Amira, L., Terman, M., 1992. Structured Interview Guide for the Hamilton Depression Rating ScaleSeasonal Affective Disorder Version (SIGH-SAD). New York State Psychiatric Institute, New York.

Young, M.A., Meaden, P.M., Fogg, L.F., Cherin, E.A., Eastman, C.I., 1997. Which environmental variables are related to the onset of seasonal affective disorder? Journal of Abnormal Psychology 106, 554-562. 